Gynäk. Rdsch. 1968;5:I-VIII

\title{
Contents, Vol. 5, 1968
}

\section{Inhaltsverzeíchnis}

Übersichtsaufsätze

Gröschel, D.: Erfahrungen mit der Kontrolle und Verhütung des Hospitalismus in einem amerikanischen Universitätskrankenhaus 1

Jantzen, K.: Physiologie und Pathologie des Fruchtwassers. Entstehung und Resorption 81

Lauritzen, Ch. : Pathogenese und Therapie der Osteoporose 161

Kurzfassungen von Zeitschriftenartikeln

Abernathy, J. R.; Greenberg, B. G.; Bradley Wells, H. und Frazier, T.M.:

Der Einfluss des Rauchens auf das Geburtsgewicht und die Schwangerschaftsdauer 220

Adams, F. H.: vide Karlberg, P.

Amiel, C; Fleurquin, N.; Larroche, J.-C. und Minkowski, A.: Über 18 Beobachtungen von Pseudomonas-Infektionen beim Neugeborenen 286

Amromin, G. D.: vide Byron, R. L.

Anderson, H. D.; McCall, K. B.; Sgouris, J. T. und Gibson, S. T. :Der klinische Gebrauch von menschlichem Trockenfibrinogen und die damit verbundenen Gefahren der Übertragung von Hepatitis143

Aoki, M.: Endometriose der Beckenlymphknoten 255

Askovitz, S. I.: vide Friedman, A. K.

Bailar, J.; Thomas, L.; Thomson, A.; Eisenberg, H. und Vick, R.: Morphologie und Überlebensrate beim Zervixkarzinom in Connecticut und Südwestengland. . 158 Ball, M. R.: vide Dagher, F. J. Barber, H. R. K. und Brunschwig, A.: Eviszeration des Beckens bei lokal fortgeschrittenen und rezidivierenden Ovarialkarzinomen 246

Barrat, J.: vide Hervet, E.

Beck, P.; Birnbaum, S. J. und Schlossberg, M.: Missgebildetes Kind nach Intrauterinpessar-Versager 262

Bellis, C. J.: Isoliertes Ileum-Segment als Harnblasenersatz nach Exenteration wegen Karzinom im Bereich des Beckens 296

Berger, S. M.: vide Friedman, A. K.

Birch, H. W.: Prämaligne Läsionen der Vulva 292

Birnbaum, S. J.: vide Beck, P.

Bjørn-Hansen, R.: Kontrastmammographie 303

Blott, G. R. und Radcliffe, R. W.: Intrauterinpessare in der Privatpraxis. Eine klinische Studie 256

Borell, U. und Fernström, I.: Die Form des kindlichen Thorax während des Durchtritts durch den Geburtskanal. Radiologische Untersuchungen 38

Bowers, W. F.: vide Leis, Ft. P.

Boyce, C. R.: vide Hodgkinson, C. P. 
Boyden, C. M.: vide Moore, F. D.

Bradley Wells, H.: vide Abernathy, J. R.

Brinkley, D. und Haybittle, J. L.: Behandlung des Brustkrebses im Stadium II 180

Brook, P. G. und Horne, H. W., Jr.: Erfahrungen mit Intrauterinpessaren bei

Frauen mit bewiesener Fertilität 260

IV

Inhaltsverzeichnis

Brown, W. E. und Allen, E. S.: Untersuchungen mit dem Intrauterinpessar . . 258

Brunschwig, A.: vide Barber, H. R. K.

Bullen, M.: vide Kincaid-Smith, P.

Bunker, J. P.: Der metabolische Effekt der Bluttransfusion 141

Burgess, J. A.: Lues-Detektion durch antenatale serologische Tests 288

Byron, R. L.; Yonemoto, R. H.; King, R. M.; Lamb, E. J.; Amromin, G. D.; Solo

mon, R. D. und Gildenhorn, V. B.: Die Behandlung eines Pseudomyxoma

peritonei nach Ruptur von Mukozelen der Appendix 228

Chapman, E.: vide Downing, G. C.

Chomiakov, Y. S. und Fedorova, R. G.: Das röntgenologische Bild der Symphysi-

tiden nach Geburten 32

Craig, I. M.: vide Heald, F. P.

Crapanzano, J. T.: vide Margulies, D.

Dagher, F. J.; Lyons, J. H.; Ball, M. R. und Moore, F. D.: Blutverlust beim

gesunden Menschen. II. Die Wirkung von Mannitol auf das Plasmavolumen und

die Verschiebung des Körperwassers nach akutem Blutverlust $\quad 58$

Dagher, F. J.: vide Moore, F. D.

Decker, W. H. und Hall, W.: Behandlung der Infektion mit Clostridium welchii

nach Fehlgeburten 107

Denoix, P.: vide Lacour, J.

Dodd, G. D.: vide Friedman, A. K.

Downing, G. C. und Chapman, E.: Rauchen und Schwangerschaft. Eine statistische

Studie über 5659 Frauen 219

Dull, H. B.: vide Mosley, J. W.

Dursi, J.: vide Leis, H. P.

Eisenberg, H.: vide Bailar, J.

Emich, J. P.: vide Zeller, R. E.

Fedorova, R. G.: vide Chomiakov, Y. S.

Fernström, I.: vide Borell, U.

Fisher, M. S.: vide Friedman, A. K.

Fletcher, G. H.: vide Zimmermann, K. W.

Fleurquin, N.: vide Amiel, C.

Frazier, T. M.: vide Abernathy, J. R.

Freda, V. J.; Gorman, J. G. und Pollack, W.: Die Verwendung von Rh-Immuno-

globulin zur Verhinderung der Rh-Erkrankung 137

Friedman, A. K.; Askovitz, S. I.; Berger, S. M.; Dodd, G. D.; Fisher, M. S.;

Lapayowker, M. S.; Moore, J. P.; Parlee, D. E.; Stein, G. N. und Pender-

grass, E. P.: Mammographie: Auswertung der in Zusammenarbeit an sieben

Uníversitätskliniken gewonnenen Erfahrungen 297 
Friedman, I. A.: vide Schewitz, L. J.

Friedman, M.: vide Ross, E. J.

Fuchs, A.-R.: vide Fuchs, F.

Fuchs, F.; Fuchs, A.-R.; Poblete, V. F., Jr., und Risk, A.: Der Effekt des Alkohols

auf die drohende Frühgeburt 104

Furuhjelm, M.: Der Einfluss der mütterlichen Berufstätigkeit auf Frühgeburt und perinatale Mortalität beí Frühgeborenen 28

Geubelle, F.: vide Karlberg, P.

Gibson, S. T.: vide Anderson, H. D.

Gildenhorn, V. B.: vide Byron, R. L.

Gorman, J. G.: vide Freda, V. J.

Greenberg, B. G.: vide Abernathy, J. R.

Hakanson, E. Y.: Traumen des weiblichen Genitales294

Inhaltsverzeichnis

$\mathrm{V}$

Hall, W.: vide Decker, W. H.

Hanley, P. H.: Die Behandlung einer mit einem Anus imperforatus kombinierten tiefsitzenden rektovaginalen Fistel 45

Haybittle, J. L.: vide Brinkley, D.

Heald, F. P.; Craig, I. M. und Ming, P. L.: Ovarialtumoren bei Jugendlichen . . 248

Hefner, J. D.: vide Hoja, W. A.

Henderson, M. und Kay, J.: Unterschiede in der Schwangerschaftsdauer bei Schwar-

zen und Weissen der niederen sozialen Schicht 97

Hervet, E. und Barrat, J.: Medizinische Indikationen zur Kontrazeption .... 47 Hodgkinson, C. P.

und Boyce, C. R.: Intraarterielle Langzeittherapie bei fortge-

schrittenen Karzinomen des Beckenraumes 196

Hoja, W. A.; Hefner, J. D. und Smith, M. R.: Asymptomatische Bakteriurie in der

Schwangerschaft 19

Horne, H. W., Jr.: vide Brook, P. G.

Hulka, B. S.: Die Früherkennung des Zervixkarzinoms bei sozial niedriggestellten

Frauen 154

Ingersoll, F. M. und Ulfelder, H.: Die Exenteration des Beckens zur Behandlung

des Zervixkarzinoms 192

Iufu, I.: vide Streja, E.

Johnson, C. E.: vide Thompson, J. P.

Johnson, J.: vide Robinson, S. C.

Jones, R. F. 111; Warren, B. L., Jr., und Thornton, W. N., Jr.: Post-partum-

Exploration von Uterus, Zervix und Vagina 130

Kajii, T.: Erfahrungen mit Thalidomid in Japan

Kalmar, Z.: Angaben zur Entwicklung von mit grosser Gewichtsdifferenz geborenen Zwillingen 111

Karlberg, P.; Adams, F. H.; Geubelle, F. und Wallgren, G.: Veränderungen des

kindlichen Thorax während der vaginalen Entbindung 37

Kaufman, R. J.: Die Behandlung des fortgeschrittenen Ovarialkarzinoms 241

Kay, J.: vide Henderson, M.

Kay, S.: Prognostische Auswertung der bei radikaler Mastektomie gefundenen 
Rotterschen Lymphknoten 188

Kincaid-Smith, P. und Bullen, M.: Bakteriurie in der Schwangerschaft

King, R. M.: vide Byron, R. L.

Krause, V. W.: vide Robinson, S. C.

Kummer, J. M. und Leavy, Z.: Verwirrung um das Gesetz zur Schwangerschaftsunterbrechung 263

Lacour, J.; Mourali, N.; Weiler, J. und Denoix, P.: Mammakarzinom und

Schwangerschaft $\quad 66$

Lamb, E. J.: vide Byron, R. L.

Lapayowker, M. S.: vide Friedman, A. K.

Larroche, J.-C.: vide Amiel, C.

Leavy, Z.: vide Kummer, J. M.

Lee, C. J.: vide Moore, F. D.

Leis, H. P.; Bowers, W. F. und Dursi, J.: Ödeme des Armes nach Mastektomie 184

Leonetti, P.-J.: vide Plasse, G.

Little, P. J.: Verhütung der Pyelonephritis gravidarum

Lyons, J. H.: vide Dagher, F. J. Lyons, J. H.: vide Moore, F. D. MacLean, L. D.: vide Shibata, H. R.

Margulies, D. und Crapanzano, J. T.: Ruptur des intakten Uterus 226

Marshall-Jones, P.: vide Ross, E. J.

VI

Inhaltsverzeichnis

McCall, K. B.: vide Anderson, H. D.

Ming, P. L.: vide Heald, F. P.

Minkowski, A.: vide Amiel, C.

Mirescu-Panaitescu, A.: vide Nicolescu, C.

Missbrauch von Hormonverordnungen in der Gynäkologie. Rundtischgespräch unter dem Vorsitz von Prof. J. Huguier 51

Moldovan, A.: vide Streja, E.

Montague, E. D.: vide Zimmermann, K. W.

Moore, J. P.: vide Friedman, A. K.

Moore, F. D.; Dagher, F. J.; Boyden, CM.; Lee, C. J. und Lyons, J. H.: Blutverlust beim gesunden Menschen. I. Verteilung und Auswirkung von Elektrolytinfusionen nach akutem Blutverlust 55

Moore, F. D.: vide Dagher, F. J.

Mosley, J. W. und Dull, H. B.: Virushepatitis nach Transfusionen 146

Mourali, N.: vide Lacour, J.

Newsham, L. S.: vide Shibata, H. R.

Nichols, G. P. und Raney, E. H.: Differentialdiagnose post partum. Herzfehler oder Fruchtwasserembolie? 131

Nicolescu, C. und Mirescu-Panaitescu, A.: Zur Infektion des Fruchtwassers ... 121

Nicoli, R.-M.: vide Plasse, G.

O'Leary, J. A.: Radikaloperation in der Schwangerschaft: Schnittentbindung mit erweiterter Uterusexstirpation 124

Paintin, D. B.: Epidemiologische Untersuchungen bei ante-partum-Blutungen.

Übersicht aller Geburten in einem Gemeindebezirk 222 
Parlee, D. E.: vide Friedman, A. K.

Pendergrass, E. P.: vide Friedman, A. K.

Plasse, G.; Nicoli, R.-M. und Leonetti, P.-J.: Die urogenitale Trichomoniase der

Frau, die Bedeutung ihrer psychosozialen Komponente 61

Poblete, V. F., Jr.: vide Fuchs, F.

Pohlman, E.: Ungewollte Konzeption. Untersuchungen über unerwünschte Wirkungen 266

Pollack, W.: vide Freda, V. J.

Pollak, V. E.: vide Schewitz, L. J.

Popescu, D. und Streza, I.: Die morphologische Grundlage der Klassifizierung von Nierengefässveränderungen während der Schwangerschaft 272

Radcliffe, R. W.: vide Blott, G. R.

Raney, E. H.: vide Nichols, G. P.

Richart, R. M.: vide Wilbanks, G. D.

Risk, A.: vide Fuchs, F.

Robinson, S. C.; Krause, V. W.; Johnson, J. und Zwicker, B.: Die Bedeutung mütterlicher bakterieller Infektionen für Infektionen und Erkrankungen des Neugeborenen 115

Ross, E. J.; Marshall-Jones, P. und Friedman, M.: Das Cushing Syndrom. Diagnostische Merkmale 200

Ross, W.: vide Shibata, H. R.

Samples, D. M. und Weed, J. C.: Gynäkologische Aspekte der idiopathischen thrombozytopenischen Purpura 290

Serment, H.: vide Vallette, C.

Sgouris, J. T.: vide Anderson, H. D.

Shibata, H. R.; Ross, W.; Newsham, L. S. und MacLean, L. D.: Diagnose der massiven Lungenembolie am Krankenbett 149

Smith, M. R.: vide Hoja, W. A.

Inhaltsverzeichnis

VII

Solomon, R. D.: vide Byron, R. L.

Schewitz, L. J.; Friedman, I. A. und Pollak, V. E.: Blutung nach Nierenbiopsie in der Schwangerschaft 275

Schlossberg, M.: vide Beck, P.

Schwarz, R. H.: vide Zeller, R. E.

Stein, G. N.: vide Friedman, A. K.

Stochen Wagenheim, H.: Nach der Sprechstunde: Wie Frauen auf eine gynäkologische Untersuchung reagieren

Streja, E.; Trifan, V.; Moldovan, A.; Tepus, D. und Iufu, I.: Beobachtungen an 290 Fallen von Herzleiden bei Schwangerschaft 118

Streza, I.: vide Popescu, D.

Tepus, D.: vide Streja, E.

Thomas, L.: vide Bailar, J.

Thompson, J. P. und Johnson, C. E.: Überlebenschancen und Geburtsleitung des zweitgeborenen Zwillings

Thomson, A.: vide Bailar, J. 
Thornton, W. N., Jr.: vide Jones, R. F. 111

Trifan, V.: vide Streja, E.

Ulfelder, H.: vide Ingersoll, F. M.

Vallette, C. und Serment, H.: Diagnose des akuten Abdomens in graviditate . . 25

Vick, R.: vide Bailar, J.

Wallgren, G.: vide Karlberg, P.

Warren, B. L., Jr.: vide Jones, R. F. 111

Watson, T. A.: Der Brustkrebs

175

Weed, J. C.: vide Samples, D. M. Weiler, J.: vide Lacour, J. Weir, D. R.: vide Weir, W. C.

Weir, W. C. und Weir, D. R.: Adoption und nachfolgende Konzeption 101

Wilbanks, G. D. und Richart, R. M.: Beziehungen zwischen dem Zustand der

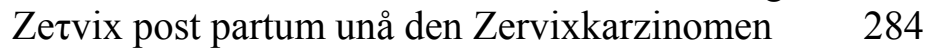

Williams, B. F. P.: Konservative Behandlung der Endometriose. Beobachtungen

nach Progestin-Therapie 250

Yonemoto, R. H.: vide Byron, R. L.

Zeller, R. E.; Schwarz, R. H. und Emich, J. P.: Uterusruptur: Eine Analyse von 43

Fallen 280

Zimmermann, K. W.; Montague, E. D. und Fletcher, G. H.: Häufigkeit, Lokalisation und Behandlung der örtlichen Rezidive von vollbehandelten Mammakarzinomen $\quad 307$

Zwicker, B.: vide Robinson, S. C.

Kasuistik

Burger, P.: Über die segmento-cervico-vaginale Atonie der Nachgeburtsperiode oder des unmittelbaren Wochenbetts 231

Korhammer, A. F.: vide Pulliam, R. R.

Pulliam, R. R. und Korhammer, A. F.: Perforation des Uterus $\quad 70$

Aus der Geschichte der Geburtshilfe

Buess, H.: Die Anfänge wissenschaftlicher Geburtshilfe im Jahre 1668: Francois

Mauriceau (1637-1709) und sein Handgriff 77

VIII

Inhaltsverzeichnis

Buess, H.: Herophilos und die Geburtshilfe in alexandrinischer Zeit

236

Varia

Einladung zu einer Wettbewerbsbeteiligung für den von der Schering AG, Berlin, gestifteten Schoeller-Junkmann-Preis 1968312 